\title{
Plasma rico em plaquetas e corticoide no tratamento da síndrome de impacto do manguito rotador: Ensaio Clínico randomizado*
}

\section{Platelet-Rich Plasma and Corticosteroid in the Treatment of Rotator Cuff Impingement Syndrome: Randomized Clinical Trial}

\footnotetext{
${ }^{1}$ Departamento de Medicina, Universidade Tiradentes, Aracaju, SE, Brasil

${ }^{2}$ Medicina Fisica e Reabilitação, Instituto de Assistência Médica ao Servidor Público Estadual (IAMSPE), São Paulo, SP, Brasil

3 Programa de pós-graduação em Saúde e meio ambiente, Universidade Tiradentes, Aracaju, SE, Brasil
}

Ronald Bispo Barreto ${ }^{1}$ Arthur Rangel Azevedo ${ }^{20}$ Mayvelise Correia de Gois ${ }^{1}$ Marianna Ribeiro de Menezes Freire ${ }^{1}$ Denison Santos Silva ${ }^{1}$ Juliana Cordeiro Cardoso ${ }^{3}$

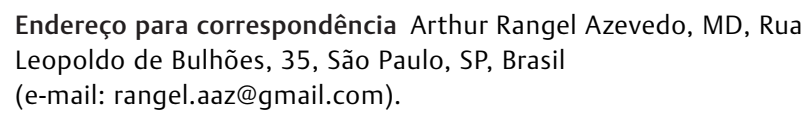

Rev Bras Ortop 2019;54:636-643.

\section{Resumo}

\section{Palavras-chave}

- plasma rico em plaquetas

- síndrome do impacto do manguito rotador

- corticosteroides

\section{Abstract}

Objetivo Analisar a eficácia do uso de plasma rico em plaquetas (PRP) no tratamento de pacientes portadores de síndrome de impacto do manguito rotador em comparação ao tratamento com injeção subacromial de corticosteroides.

Métodos $\mathrm{O}$ estudo é de caráter comparativo, longitudinal, duplo cego e randomizado. A evolução clínica dos pacientes foi quantificada pelas escalas The Disabilities of the Arm, Shoulder and Hand (DASH) outcome measure, University of California Los Angeles (UCLA) shoulder rating scale e Constant-Murley shoulder outcome score (CMS) no dia da aplicação, e novamente após 1, 3, e 6 meses.

Resultados Não foram encontradas diferenças etsatisticamente significativas $(p<0.05)$ ao comparar os resultados do DASH outcome measure, UCLA shoulder rating scale, e CMS dos dois grupos na admissão. Após o tratamento, ambos os grupos apresentaram melhora significativa tanto do DASH, quanto do UCLA $(p<0,05)$. Entretanto, o escore do CMS referente ao tratamento com corticoide mostrou-se pior no $6^{\circ}$ mês em comparação com o escore à admissão.

Conclusão Esses achados sugerem que o PRP é um tratamento seguro e que pode ser uma ferramenta útil no arsenal terapêutico contra doenças do manguito rotador, uma vez que não foram encontradas diferenças significativas entre os grupos que receberam PRP e injeção subacromial de corticosteroides.

Objective This research aims to study the effectiveness of platelet-rich plasma (PRP) in the treatment of patients with rotator cuff impingement syndrome compared with the treatment with subacromial injection of corticosteroids.

\footnotetext{
* Trabalho feito no DeCós Day Hospital, Aracaju, SE, Brasil. Originalmente Publicado por Elsevier Editora Ltda.
}

recebido

12 de Novembro de 2017 aceito

27 de Fevereiro de 2018
DOI https://doi.org/

10.1016/j.rboe.2018.03.002 ISSN $0102-3616$
Copyright $(2019$ by Sociedade Brasileira License terms de Ortopedia e Traumatologia. Published by Thieme Revinter Publicações Ltda, Rio de Janeiro, Brazil 


\section{Keywords \\ - platelet-rich plasma \\ - shoulder impingement syndrome \\ - adrenal cortex hormones}

Methods This is a double-blind, randomized, comparative clinical trial. The patients were clinically evaluated with the use of the Disabilities of the Arm, Shoulder and Hand (DASH) outcome measure, University of California Los Angeles (UCLA) shoulder rating scale and Constant-Murley shoulder outcome score (CMS) on the day of application, and then again after 1,3 , and 6 months.

Results No statistically significant differences were found $(p<0.05)$ when comparing the results of the DASH outcome measure, UCLA shoulder rating scale and CMS of the two groups at baseline and after 1, 3, and 6 months of treatment with subacromial injection. After the treatment, both groups showed a significant improvement in the DASH and UCLA scores $(p<0.05)$ when compared with the baseline. However, the CMS at 6 months after treatment with steroids trea were lower than the baseline.

Conclusions These findings suggest that PRP is a safe treatment and can be a useful tool in the therapeutic arsenal against of the rotator cuff diseases for there was no significant difference between the subacromial use of PRP and corticosteroids.

\section{Introdução}

A cronicidade das manifestações clínicas da síndrome de impacto do manguito rotador faz com que novas terapias que ajudem a melhorar o manejo da dor sejam necessárias.

O plasma rico em plaquetas (PRP) é uma modalidade terapêutica em evolução que vem ganhando destaque na atenção primária, na reabilitação, e na medicina esportiva. 0 PRP é uma solução concentrada de plaquetas preparada com sangue autólogo, o que torna este tratamento seguro para uso clínico. ${ }^{1}$ O PRP modula fatores bioativos na região da lesão, aumentando o potencial de regeneração. ${ }^{2}$ O PRP aumenta a produção de colágeno tipo I, a proliferação de tenócitos, e a síntese de fator transformador do crescimento (TGF, na sigla em inglês) $\beta 1$, fator de crescimento derivado de plaquetas (PDGF, na sigla em inglês), fator de crescimento endotelial vascular (VEGF, na sigla em inglês) e fator de crescimento epidérmico (EGF, na sigla em inglês), além de estimular a síntese da matriz ligamentar. ${ }^{3-6}$

As injeções de corticosteroides são comumente administradas em lesões tendíneas. ${ }^{7}$ A aplicação subacromial é considerada uma opção terapêutica barata e eficaz, mas os efeitos colaterais dos corticosteroides são preocupantes na prática clínica. Além disso, rupturas tendíneas foram observadas após injeções locais de corticosteroides, em casos de repetição do tratamento ou mesmo depois de uma única aplicação. ${ }^{8}$ Outras complicações não infecciosas também podem ocorrer, como atrofia de nervos, hipopigmentação cutânea, calcificação distrófica ao redor da cápsula articular, hiperglicemia e inibição do eixo hipotalâmico-hipofisário por 1 a 2 semanas após a administração. ${ }^{9}$

A literatura atual sobre o PRP como uma nova modalidade terapêutica é limitada, devido à ausência de padronização dos protocolos de estudo, das técnicas de preparo do PRP e dos métodos de medida da melhora clínica; portanto, novas pesquisas precisam ser realizadas para o entendimento concreto dessa modalidade terapêutica.

O objetivo deste estudo é analisar a eficácia terapêutica da infiltração subacromial de PRP em pacientes com síndrome de impacto do manguito rotador em comparação à injeção subacromial de corticosteroide.

\section{Métodos}

Este é um estudo clínico intervencionista, duplo-cego, randomizado e controlado, em pacientes com síndrome de impacto do manguito rotador. Os pacientes foram randomizados em blocos para recebimento do tratamento com PRP ou corticosteroide injetável. A amostra foi composta por 51 pacientes, sendo 25 no grupo tratado com corticosteroide e 26 no grupo tratado com PRP. Estima-se que o número amostral de pacientes tenha um intervalo de confiança de $95 \%$ e poder de $80 \%$ para deteç̧ão de uma diferença de 15 pontos no escore DASH, com desvio-padrão de 10 a 20,4 em cada grupo. Para permitir a saída de aproximadamente $20 \%$ dos pacientes, um número conservador, recrutamos um total de 51 indivíduos. Não houve instituição de protocolo de imputação em relação à perda de amostras. A randomização em bloco foi realizada com o mecanismo online sealedenvelope.com, que gerou uma lista do número de admissão do paciente e o tratamento correspondente. 0 pesquisador responsável pela admissão do paciente não estava ciente da randomização ou alocação do grupo; além disso, a ordem de admissão foi cuidadosamente seguida.

O PRP foi obtido a partir de $15 \mathrm{~mL}$ de sangue periférico do paciente; essa alíquota foi submetida a um protocolo de dupla centrifugação (1.500 rpm por 6 minutos, depois a 3.500 rpm por 15 minutos em uma macrocentrífuga Coleman 80-2C) e separação manual de plaquetas com micropipetas, inclusive da camada leucoplaquetária, e sua transferência para um tubo de ensaio para concentração. Isso gera cerca de $3 \mathrm{~mL}$ de PRP, que são transferidos para a seringa para injeção. Todos os materiais utilizados eram estéreis e descartáveis. O corticosteroide administrado foi o dipropionato de betametasona. Para manter a característica duplo-cega do estudo, todos os pacientes foram submetidos à coleta de sangue periférico, e a natureza do tratamento foi ocultada do paciente e do profissional de saúde. Um dos pesquisadores foi responsável pela alocação dos pacientes, preparo do tratamento, e garantia do duplo-cegamento. As 
injeções foram feitas no espaço subacromial por meio de abordagem posterior após a anestesia com lidocaína a $1 \%$ e não foram guiadas por ultrassonografia. Todos os participantes foram instruídos a repousar o ombro injetado por 24 horas. Alterações na medicação ou no programa de reabilitação não foram recomendadas.

Para avaliar a resposta terapêutica, as ferramentas utilizadas foram a ascala de classificação do ombro da UCLA (UCLA Shoulder Rating Scale), o escore de ombro de ConstantMurley (Constant-Murley Shoulder Outcome Score), e a medida de desfecho DASH (Disabilities of the Arm, Shoulder and Hand). Os pacientes foram avaliados antes do procedimento e 1, 3, e 6 meses após a intervenção. Todos os pacientes foram questionados sobre possíveis efeitos colaterais durante o período de acompanhamento.

Os critérios de inclusão foram: idade entre 18 e 70 anos, resultado positivo ao teste de Neer, nenhum tratamento prévio com corticosteroides intravenosos, injetáveis ou orais nos últimos 12 meses, concentração de hemoglobina maior que $11 \mathrm{~g} / \mathrm{dL}$, contagem de plaquetas maior que $150.000 / \mathrm{mm}^{3}$, e assinatura do termo de consentimento livre e esclarecido. Os critérios de exclusão foram: associação à capsulite adesiva, ruptura completa do manguito rotador à ultrassonografia (sendo que todas as outras formas de tendinopatia foram incluídas) e uso de corticosteroides orais ou parenterais durante o estudo.

Esta pesquisa foi totalmente realizada no deCós Day Hospital em Aracaju, Brasil. O recrutamento e o acompanhamento dos pacientes foram realizados entre outubro de
2015 e julho de 2016 e terminaram após 6 meses de acompanhamento do último paciente recrutado. Os dados foram tabulados e analisados usando o programa Statistical Package for the Social Sciences (SPSS), versão 19.0 (IBM Corp., Armonk, NY, EUA).

Nesta pesquisa, o sigilo e a privacidade dos profissionais participantes foram assegurados, garantindo que as informações do questionário não fossem utilizadas em detrimento das pessoas envolvidas. Todos os pacientes assinaram o termo de consentimento livre e esclarecido. Este estudo foi aprovado pelo comitê de ética em pesquisa da Universidade Tiradentes CEP/UNIT (CAAE: 49144515.90000.5371) e está registrado no Registro Brasileiro de Ensaios Clínicos - ReBEC (UTN: U11111195-9769).

\section{Resultados}

A idade média dos pacientes desta amostra foi de 53 anos; $64 \%$ dos pacientes eram do sexo feminino e apresentavam dor no ombro direito (45\%), peso médio de $72 \mathrm{~kg}, 1,6 \mathrm{~m}$ de altura e índice de massa corporal (IMC) de $28 \mathrm{~kg} / \mathrm{m}^{2}$. O perfil epidemiológico da amostra pode ser observado na - Tabela 1. Dos pacientes estudados, apenas 40 retornaram para avaliação após 1 mês de tratamento (19 no grupo tratado com corticosteroide e 21 no grupo tratado com PRP), 27 completaram 3 meses de tratamento (10 no grupo tratado com corticosteroide e 17 no grupo tratado com PRP) e 22 os pacientes completaram 6 meses de tratamento (10 no grupo tratado com corticosteroide e 12 no

Tabela 1 Perfil epidemiológico dos pacientes com síndrome de impacto do manguito rotador.

\begin{tabular}{|c|c|c|c|c|}
\hline & \multicolumn{2}{|c|}{ Tratamento recebido } & \multirow[b]{2}{*}{ Total } & \multirow[b]{2}{*}{ Valor de $p$} \\
\hline & PRP & Corticoide & & \\
\hline \multicolumn{5}{|l|}{ Idade - n (\%) } \\
\hline$<35$ anos & $1(4)$ & $1(4)$ & $2(4)$ & 0,41 \\
\hline $36-45$ anos & $4(15)$ & $4(16)$ & $8(16)$ & \\
\hline $46-55$ anos & $9(35)$ & $8(32)$ & $17(33)$ & \\
\hline $56-65$ anos & $10(38)$ & $6(24)$ & $16(31)$ & \\
\hline$>65$ anos & $2(8)$ & $2(8)$ & $4(8)$ & \\
\hline Não informada & $0(0)$ & $4(16)$ & $4(8)$ & \\
\hline Total & $26(49)$ & $25(51)$ & $51(100)$ & \\
\hline Média \pm DP & $53,2 \pm 9,4$ & $53 \pm 11$ & $53,1 \pm 10$ & \\
\hline \multicolumn{5}{|l|}{ Sexo - n (\%) } \\
\hline Masculino & $11(42)$ & $7(28)$ & $18(36)$ & 0,433 \\
\hline Feminino & $15(57)$ & $18(72)$ & $33(64)$ & \\
\hline \multicolumn{5}{|c|}{ Lado acometido - n (\%) } \\
\hline Ombro direito & $11(44)$ & $21(80)$ & $23(45)$ & 0,015 \\
\hline Ombro esquerdo & $11(44)$ & $5(20)$ & $16(31)$ & \\
\hline \multicolumn{5}{|c|}{ Dados antropométricos - Média \pm DP } \\
\hline Altura (m) & $1,6 \pm 0,1$ & $1,6 \pm 0,1$ & $1,6 \pm 0,1$ & 0,054 \\
\hline Peso $(\mathrm{kg})$ & $72,2 \pm 13,7$ & $71,8 \pm 16,3$ & $72,0 \pm 14,7$ & 0,196 \\
\hline IMC $\left(\mathrm{kg} / \mathrm{m}^{2}\right)$ & $28,4 \pm 3,7$ & $27,6 \pm 5,0$ & $28,0 \pm 4,3$ & 0,258 \\
\hline
\end{tabular}

Abreviaturas: DP, desvio padrão; IMC, índice de massa corporal; PRP, plasma rico em plaquetas. 
Tabela 2 Comparação da eficácia do tratamento da tendinopatia do manguito rotador - plasma rico em plaquetas (PRP) versus corticoide.

\begin{tabular}{|l|l|l|l|l|}
\hline & \multicolumn{2}{|l|}{$\begin{array}{l}\text { Tratamento } \\
\text { recebido }\end{array}$} & & \\
\cline { 2 - 5 } & PRP & Corticoide & IC 95\% & $\begin{array}{l}\text { Valor } \\
\text { de } p\end{array}$ \\
\hline $\begin{array}{l}\text { Medida de } \\
\text { desfecho DASH }\end{array}$ & & & \\
\hline Admissão & 53,3 & 54,5 & {$[-52,9 ; 21,35]$} & 0,135 \\
\hline 1 mês & 32,5 & 22,9 & {$[-37,01 ;-0,85]$} & 0,205 \\
\hline 3 meses & 23,5 & 20,2 & {$[-104,76 ; 44,08]$} & 0,235 \\
\hline 6 meses & 21,6 & 17,7 & {$[-14,14 ; 18,57]$} & 0,824 \\
\hline Escala de classificação do ombro da UCLA & \\
\hline Admissão & 13,4 & 14,9 & {$[-3,02 ; 6,19]$} & 0,926 \\
\hline 1 mês & 23 & 23,9 & {$[-4,45 ; 6,07]$} & 0,832 \\
\hline 3 meses & 29,3 & 29,6 & {$[-9,54 ; 10,16]$} & 0,898 \\
\hline 6 meses & 27 & 27,6 & {$[-8,53 ; 9,73]$} & 0,005 \\
\hline Escore de ombro de Constant-Murley & \\
\hline Admissão & 45,9 & 49,8 & {$[-5,04 ; 12,80]$} & 0,049 \\
\hline 1 mês & 55,4 & 58,8 & {$[-4,43 ; 11,12]$} & 0,503 \\
\hline 3 meses & 59,8 & 68 & {$[-5,39 ; 21,74]$} & 0,848 \\
\hline 6 meses & 62,9 & 56,9 & {$[-21,45 ; 9,42]$} & 0,228 \\
\hline
\end{tabular}

Abreviaturas: DASH, Disabilities of the Arm, Shoulder and Hand; IC, intervalo de confiança; PRP, plasma rico em plaquetas; UCLA, University of California Los Angeles.

grupo tratado com PRP). Nenhum protocolo de imputação foi instituído neste estudo, e todos os dados obtidos foram utilizados na análise.
Os valores obtidos por meio dos questionários no momento anterior à intervenção e depois de 1, 3, e 6 meses foram comparados entre os grupos pelo teste $t$ de Student independente e Mann-Whitney e são apresentados na - Tabela 2.

Ao comparar os resultados da medida de desfecho DASH após 1, 3, e 6 meses de tratamento com os valores obtidos antes da intervenção, identificamos uma diferença estatisticamente significativa $(p<0,05)$ nos grupos tratados com PRP e corticosteroide em todos os momentos estudados, como mostra a - Fig. 1. À avaliação da progressão da escala de classificação do ombro da UCLA (-Fig. 2), também identificamos diferenças estatisticamente significativas $(p<0,05)$ na comparação das pontuações obtidas à admissão e após $1 \mathrm{e}$ 3 meses de tratamento nos 2 grupos.

No escore de ombro de Constant-Murley, a diferença entre os valores obtidos durante o acompanhamento do grupo tratado com PRP foi estatisticamente significativa após um mês $(p<0,05)$, mas não foi significativa aos $3(p=0,07)$ e aos 6 meses ( $p=0,103)$ em comparação aos valores obtidos à admissão, como mostra a - Fig. 3. O grupo tratado com corticosteroide apresentou uma diferença significativa após 1 e 3 meses de tratamento $(p<0,05)$. No entanto, não houve diferença significativa após 6 meses ( $p=0,760)$ em comparação ao valor obtidoà admissão. A avaliação estatística da progressão entre os grupos de tratamento foi realizada por meio da aplicação do teste $t$ de Student com amostras pareadas e do teste de Wilcoxon.

\section{Discussão}

Este estudo longitudinal, duplo-cego, controlado e randomizado demonstra os efeitos da infiltração única de corticosteroides e PRP no tratamento da tendinopatia do manguito rotador.

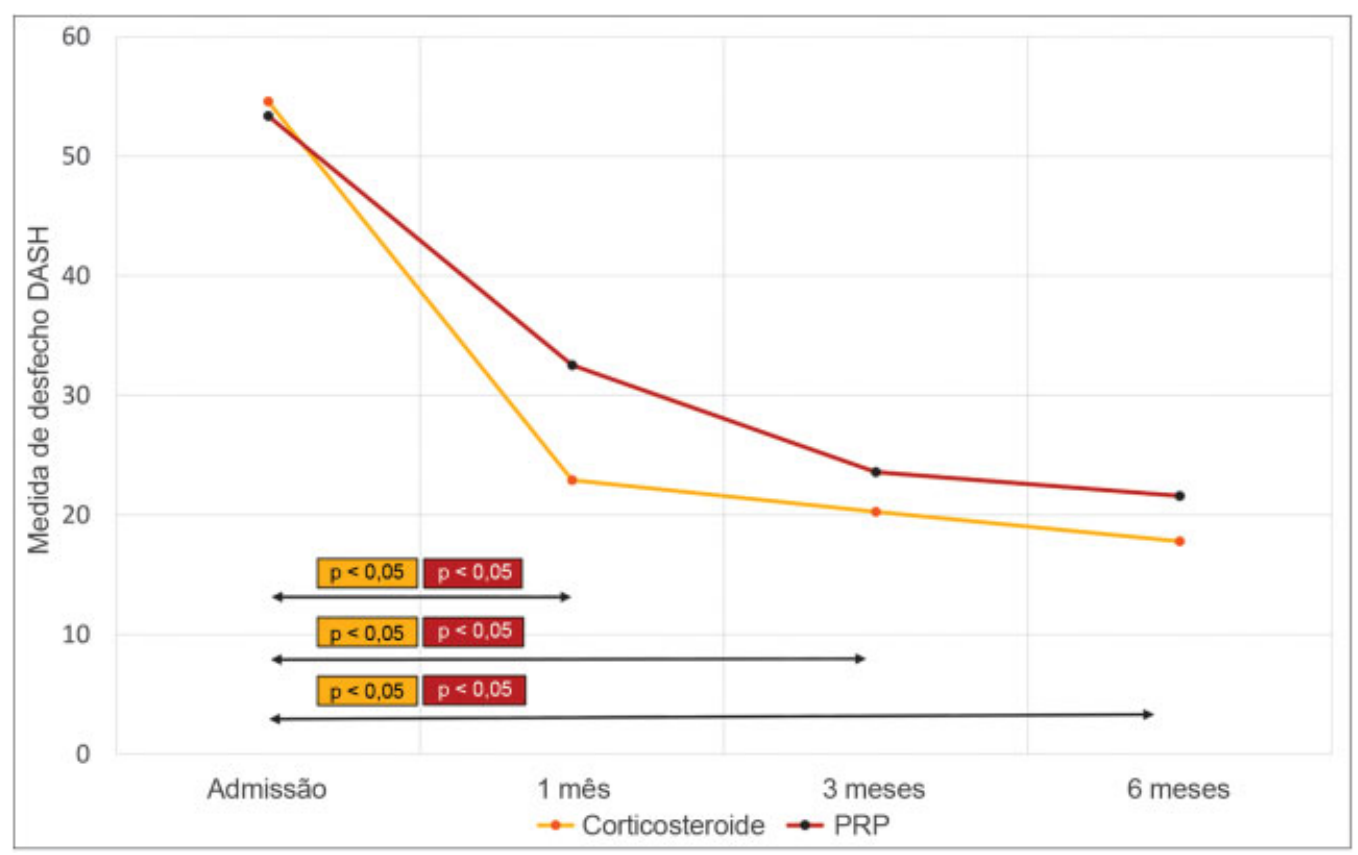

Fig. 1 Evolução da pontuação da medida de desfecho Disabilities of the Arm, Shoulder and Hand (DASH) em pacientes com tendinopatia do manguito rotador após 1, 3, e 6 meses de tratamento com corticosteroides e plasma rico em plaquetas (PRP). (O valor de $p$ mostrado representa a diferença estatística em comparação ao valor obtido à admissão). 


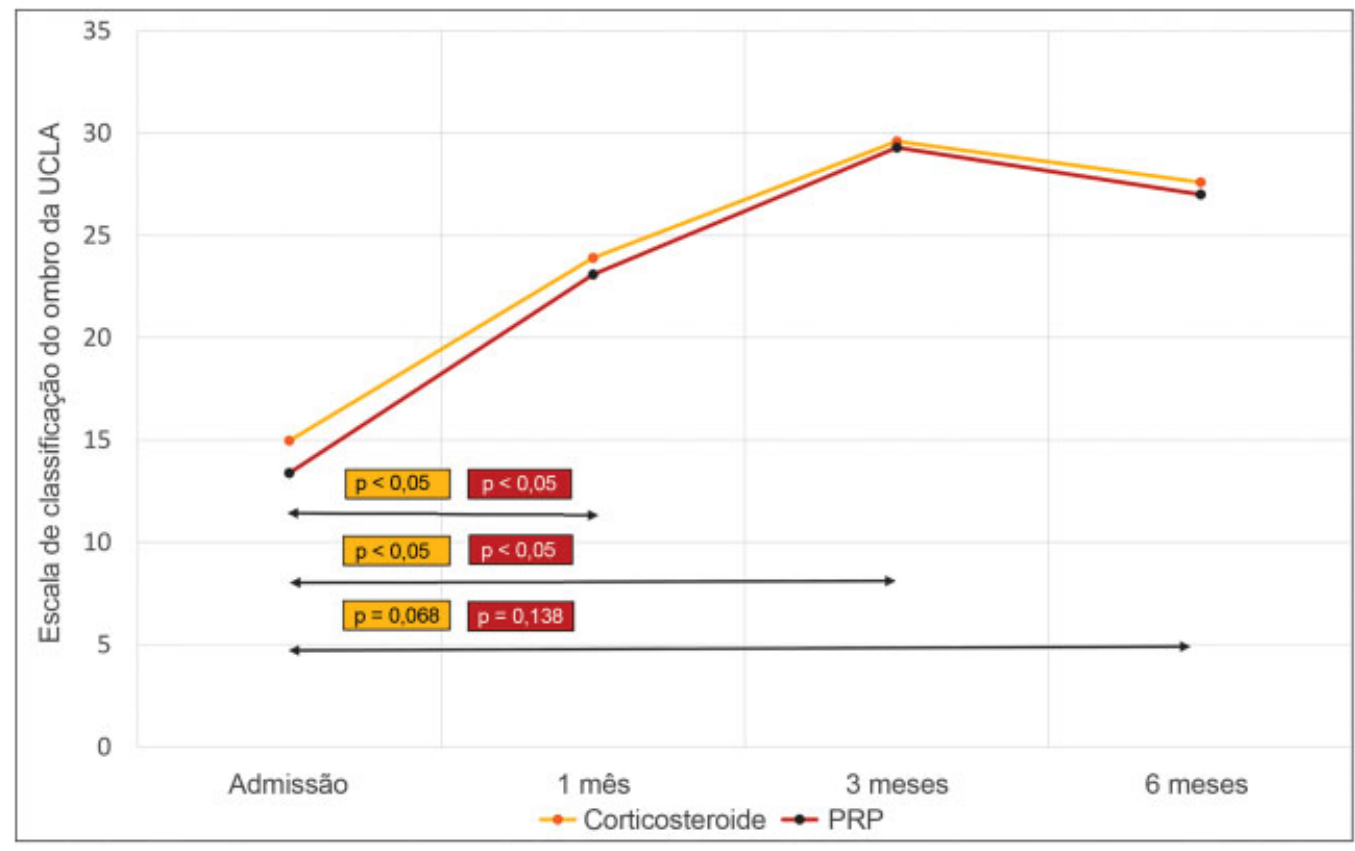

Fig. 2 Evolução da escala de classificação do ombro UCLA em pacientes com tendinopatia do manguito rotador após 1, 3, e 6 meses de tratamento com corticosteroides e plasma rico em plaquetas (PRP). (O valor de $p$ mostrado representa a diferença estatística em comparação ao valor obtido à admissão).

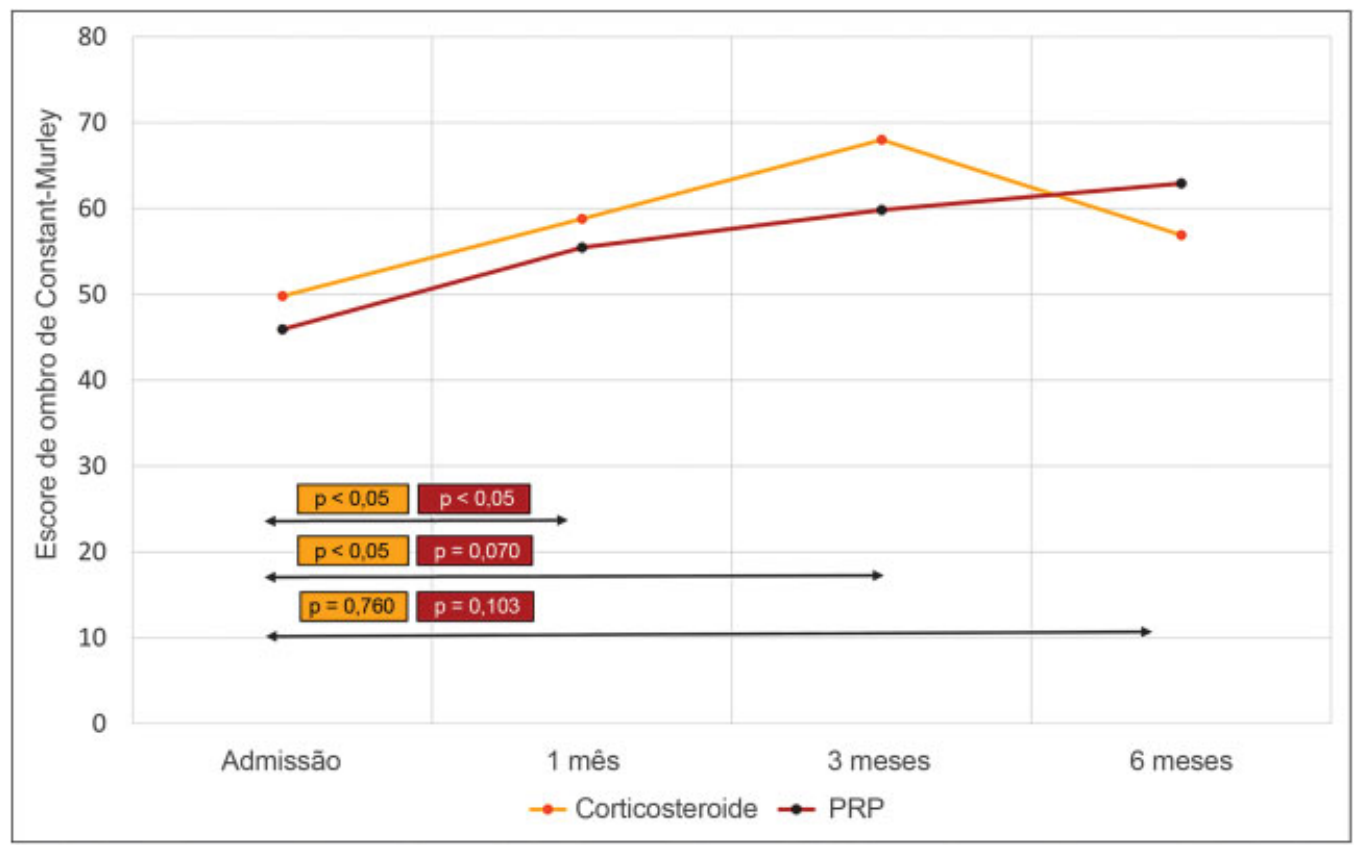

Fig. 3 Evolução do escore do ombro de Constant-Murley em pacientes com tendinopatia do manguito rotador após 1, 3, e 6 meses de tratamento com corticosteroides e plasma rico em plaquetas (PRP). (O valor de $p$ mostrado representa a diferença estatística em comparação ao valor obtido à admissão).

Nesse estudo, os pacientes que receberam PRP apresentaram uma diferença média de 20,8 pontos no escore DASH durante o $1^{\circ}$ mês de acompanhamento, o que já mostra uma melhora significativa na dor e na função dos membros em curto prazo (diferença superior a 15 pontos). ${ }^{10}$ Por outro lado, os pacientes do grupo tratado com corticosteroide apresentaram diferença média de 31,6 pontos, o que mostra que ambos os grupos apresentaram uma redução estatisticamente significativa $(p<0,05)$ do escore DASH em curto prazo. Essas pontuações continuaram significativas em comparação aos valores obtidos à admissão mesmo após 3 e 6 meses de acompanhamento.

O grupo de pacientes tratados com corticosteroides aparentemente apresentou melhoria na resposta à dor e na função após 1 e 3 meses de tratamento. Aos 6 meses de acompanhamento, os dois grupos apresentaram resultados semelhantes. No entanto, as diferenças apresentadas entre os grupos nos 3 momentos não foram estatisticamente significativas 
$(p>0,05)$. Os resultados positivos e significativos da medida de desfecho DASH simbolizam que ambas as modalidades terapêuticas provocaram melhora nos desafios diários associados ao ombro doloroso segundo a percepção do próprio paciente, bem como diminuição dos sintomas e menor influência psicossocial e ocupacional desses sintomas.

A melhora clínica observada no grupo tratado com PRP neste estudo também foi observada por outros autores. Um estudo longitudinal ${ }^{11}$ sobre o uso de PRP na tendinopatia do manguito rotador identificou uma melhora gradual na escala analítica visual após 8 e 52 semanas de tratamento. Os autores utilizaram uma forma diferente para medida da resposta terapêutica, mas notaram uma redução gradual dos sintomas, um achado também observado em nossa pesquisa e caracterizado pelos valores decrescentes no escore DASH.

As outras pontuações usadas neste estudo tiveram resultados semelhantes. A escala de classificação de ombro da UCLA associa variáveis subjetivas de dor e função a variáveis objetivas da função do ombro para avaliação da resposta terapêutica depois de um procedimento através de parâmetros de dor, função, movimento, força e satisfação do paciente. A resposta terapêutica foi significativa após 1 e 3 meses de tratamento $(p<0,05)$, mas a diferença em comparação aos valores obtidos à admissão não foi significativa aos 6 meses $(p=0,138)$, o que pode ser explicado pelo baixo número de participantes que terminaram o estudo. Um estudo ${ }^{12}$ usou PRP intraoperatório em reparos artroscópicos de rupturas completas do manguito rotador e descobriu que o PRP gerava resultados positivos nos escores de Constant-Murley e da UCLA.

O escore de ombro de Constant-Murley é um questionário mais objetivo que mede a função do ombro por meio de sua amplitude de movimento e força, com menor relevância dos sintomas na pontuação final. Os resultados do escore de Constant-Murley mostram que uma melhora mensurável na função do ombro foi identificada apenas no $1^{\circ}$ mês após o tratamento com PRP e até 3 meses após o uso de corticosteroide e que, neste último, houve uma piora geral da função em relação à admissão. Em comparação aos resultados de outro estudo $^{12}$, os achados dessa pesquisa parecem menos significativos, mas esse fato provavelmente se deve à associação do tratamento cirúrgico à administração de PRP. O PRP ${ }^{13}$ melhorou vários parâmetros do escore de Constant-Murley, incluindo dor e amplitude de movimento, até 3 meses após a administração, com resultados semelhantes aos observados no presente estudo.

Uma meta-análise sistemática de revisão ${ }^{14}$ estudou a eficácia de PRP na cicatrização de tendões e ligamentos em 37 artigos. Neste estudo, os grupos tratados com PRP apresentaram significativamente menos dor no acompanhamento em longo prazo quando comparados aos grupos controles. Esse achado pode ser correlacionar a nosso estudo ao considerar a piora em longo prazo dos escores de Constant-Murley no grupo tratado com corticosteroide, enquanto os efeitos do PRP tiveram maior durabilidade.

Um estudo controlado randomizado prospectivo ${ }^{15}$ compararam a injeção de PRP e corticosteroide em 40 pacientes com ruptura parcial do manguito rotador. Ambos os grupos apresentaram melhora estatisticamente significativa das pontuações em comparação aos valores obtidos antes da administração. Na comparação entre grupos, o tratamento com PRP teve resultados significativamente melhores às 12 semanas, mas sem diferença depois de 6 meses.

Por outro lado, um estudo comparativo ${ }^{16}$ sobre o uso de PRP e prednisona no tratamento da síndrome de manguito rotador concluiu que, embora ambos tenham tido resultados positivos, a resposta clínica dos pacientes que receberam corticosteroides foi significativamente melhor em comparação àqueles tratados com PRP, tanto às 6 semanas quanto aos 6 meses. 0 presente estudo concorda com os dois estudos ao mostrar resultados positivos com ambos os tratamentos; no entanto, nossos achados concordam com Shams et al. ao considerar a administração de PRP equivalente ou não inferior em comparação às injeções de corticosteroides. Este estudo concorda com os dados de Say et al. em relação à resposta em curto prazo, mas discorda na resposta em longo prazo, pois a diferença em seis meses entre os dois grupos não foi significativa. Essa divergência tem sido predominante na literatura sobre PRP e esses resultados variados podem ser decorrentes dos diferentes protocolos de preparo, do corticosteroide utilizado ou da metodologia do estudo. Novos estudos ainda são necessários para maior compreensão.

Um estudo clínico controlado, duplo-cego e randomizado ${ }^{17}$ utilizou PRP no tratamento da tendinopatia crônica do manguito rotador. Os autores observaram que não houve diferença significativa entre o uso de PRP e placebo nos escores Western Ontario Rotator Cuff(WORC) index, Shoulder Pain and Disability Index (SPADI) e escala visual análoga (EVA) ao longo do estudo, concluindo que o PRP não foi mais eficaz na melhoria da qualidade de vida, da dor, da disfunção ou da amplitude de movimento do que o placebo . Este estudo se opõe ao uso do PRP no tratamento dessa doença, com nível 1 de evidência. Isso apoia a hipótese de que os corticosteroides podem não ter valor no tratamento em longo prazo das tendinopatias do manguito rotador, uma vez que, através da comparação realizada neste estudo, não houve diferença significativa entre as respostas terapêuticas geradas por corticosteroides e PRP. Além disso, os autores identificaram uma melhora terapêutica nos dois grupos estudados e acreditam que também seria observada se houvesse um grupo não submetido a procedimento algum, uma vez que apenas o exercício físico já causaria melhora das tendinopatias do manguito rotador em curto e longo prazo. ${ }^{18}$

A aparente melhora dos pacientes submetidos a infiltrações subacromiais também pode estar associada ao agulhamento seco realizado para manter o caráter duplo-cego da pesquisa. ${ }^{19}$ Os autores identificaram um resultado positivo inesperado em pacientes submetidos ao agulhamento seco, explicado pelo estímulo à regeneração por extravasamento de sangue e subsequente reação inflamatória local, que, embora limitada, é um mecanismo semelhante ao do PRP.

Apesar dos resultados positivos com o uso subacromial de corticosteroides, as evidências da literatura não valorizam o uso desse medicamento em doenças do manguito rotador. Uma revisão ${ }^{20}$ identificou dois estudos que favoreceram o uso de corticosteroides, outros dois estudos em que não houve diferença significativa entre a administração de corticosteroide 
e placebo, e um estudo que favoreceu os resultados do placebo. Além disso, há estudos ${ }^{20,21}$ que analisaram a associação de corticosteroides com antiinflamatórios não-esteroides (AINEs) em comparação à administração isolada de AINEs e não observaram diferenças significativas entre os dois grupos; isso sugere que respostas terapêuticas semelhantes ou melhores podem ser obtidas sem expor o paciente aos possíveis efeitos colaterais das injeções de corticosteroides.

Os pacientes submetidos a injeções de corticosteroides tiveram que procurar serviços de saúde com mais frequência durante 1 ano de acompanhamento devido à persistência ou recidiva dos sintomas do que pacientes que fizeram apenas fisioterapia motora, com necessidade de repetição das injeções. ${ }^{22}$ Esses achados são comparáveis aos observados no presente estudo na avaliação de 6 meses do escore de Constant-Murley e da escala da UCLA, uma vez que houve diminuição dessas pontuações, o que simboliza a piora dos sintomas em relação à avaliação anterior e pode indicar a necessidade de novas aplicações ou de outra terapia adjuvante.

Por seu caráter autólogo, o PRP é um tratamento seguro para uso clínico. ${ }^{1}$ Os pacientes deste estudo não relataram nenhum efeito colateral durante todo o período de acompanhamento além de dor local até 24 horas após a injeção, o que é esperado devido ao término do efeito do anestésico local.

As limitações deste estudo foram: a ausência de um grupo placebo para comparação à história natural da doença e a ausência de acompanhamento completo de 6 meses de todos os pacientes da amostra. Este trabalho ainda abre a possibilidade de otimização, com protocolos de preparo de PRP, melhores concentrações e reprodutibilidade. Os estudos atuais parecem indicar que várias aplicações de PRP podem ajudar a aumentar a resposta clínica a esse tratamento. A aplicação de três doses de PRP demonstrou ser significativa no tratamento dos primeiros estágios da osteoartrite ${ }^{23} \mathrm{e}$ da tendinopatia patelar crônica ${ }^{24}$ e esse padrão também pode ser repetido nas tendinopatias do manguito rotador. Além disso, o PRP foi estudado ${ }^{25}$ em associação ao corticosteroide, já que não altera os efeitos da dexametasona sobre os tenócitos ou sua ação antiinflamatória, demonstrando que esses tratamentos podem gerar uma resposta clínica sinérgica em relação à dor e regeneração tecidual.

A eficácia do PRP como modalidade terapêutica ainda precisa ser mais esclarecida, mas suas características o tornam promissor no tratamento da tendinopatia do manguito rotador. Os corticosteroides, embora bem estudados quanto a sua eficácia e efeitos colaterais, continuam sendo uma preocupação crescente em seu uso crônico na prática clínica.

\section{Conclusão}

Este estudo conclui que as injeções subacromiais de plasma autólogo rico em plaquetas e corticosteroides têm resposta clínica positiva e semelhante no tratamento de tendinopatias do manguito rotador. Ao analisar as pontuações que consideram a amplitude de movimento e força, os pacientes apenas apresentaram resultados estatisticamente significativos em curto prazo (1-3 meses), e o grupo tratado com corticosteroide sofreu piora funcional aos 6 meses. Esses achados sugerem que o PRP é um tratamento seguro e pode ser uma boa ferramenta no arsenal terapêutico contra doenças do manguito rotador, principalmente ao evitar os possíveis efeitos adversos associados ao uso de corticosteroides.

Conflitos de Interesse

Os autores declaram não haver conflitos de interesse.

\section{Referências}

1 Killian ML, Cavinatto L, Galatz LM, Thomopoulos S. Recent advances in shoulder research. Arthritis Res Ther 2012;14(03):214

2 Molloy T, Wang Y, Murrell G. The roles of growth factors in tendon and ligament healing. Sports Med 2003;33(05):381-394

3 Klein MB, Yalamanchi N, Pham H, Longaker MT, Chang J. Flexor tendon healing in vitro: effects of TGF-beta on tendon cell collagen production. J Hand Surg Am 2002;27(04):615-620

4 Lynch SE, Nixon JC, Colvin RB, Antoniades HN. Role of plateletderived growth factor in wound healing: synergistic effects with other growth factors. Proc Natl Acad Sci U S A 1987;84(21): 7696-7700

5 Varga J, Jimenez SA. Stimulation of normal human fibroblast collagen production and processing by transforming growth factor-beta. Biochem Biophys Res Commun 1986;138(02):974-980

6 Zhang F, Liu H, Stile F, et al. Effect of vascular endothelial growth factor on rat Achilles tendon healing. Plast Reconstr Surg 2003; 112(06):1613-1619

7 Rees JD, Wilson AM, Wolman RL. Current concepts in the management of tendon disorders. Rheumatology (Oxford) 2006;45 (05):508-521

8 Kleinman M, Gross AE. Achilles tendon rupture following steroid injection. Report of three cases. J Bone Joint Surg Am 1983;65(09): 1345-1347

9 Roberts WN Jr. Joint aspiration or injection in adults: Technique and indications. In: UpToDate, Post TW, edotors, UpToDate, Waltham, MA. (acessado em: 21 de out. 2016).

10 Beaton DE, Davis AM, Hudak P, McConnell S. The DASH (Disabilities of the Arm, Shoulder and Hand) outcome measure: What do we know about it now? Br J Hand Ther 2001;6(04):109-118

11 Scarpone M, Rabago D, Snell E, et al. Effectiveness of platelet-rich plasma injection for rotator cuff tendinopathy: a prospective open-label study. Glob Adv Health Med 2013;2(02):26-31

12 Malavolta EA, Gracitelli ME, Sunada EE, et al. Platelet-rich plasma in arthroscopic repairs of complete tears of the rotator cuff. Rev Bras Ortop 2015;47(06):741-747

13 Tahririan MA, Moezi M, Motififard M, Nemati M, Nemati A. Ultrasound guided platelet-rich plasma injection for the treatment of rotator cuff tendinopathy. Adv Biomed Res [Internet]. 27 de dezembro de 2016. Disponível em: https://www.ncbi.nlm. nih.gov/pmc/articles/PMC5220679/

14 Chen X, Jones IA, Park C, Vangsness CT. The Efficacy of PlateletRich Plasma on Tendon and Ligament Healing: A Systematic Review and Meta-analysis With Bias Assessment. Am J Sports Med. 1 o de dezembro de 2017;363546517743746.

15 Shams A, El-Sayed M, Gamal O, Ewes W. Subacromial injection of autologous platelet-rich plasma versus corticosteroid for the treatment of symptomatic partial rotator cuff tears. Eur J Orthop Surg Traumatol 2016;26(08):837-842

16 Say F, Gurler D, Bulbul M. Platelet-rich plasma versus steroid injection for subacromial impingement syndrome. J Orthop Surg (Hong Kong) 2016;24(01):62-66

17 Kesikburun S, Tan AK, Yilmaz B, Yaşar E, Yazicioğlu K. Platelet-rich plasma injections in the treatment of chronic rotator cuff tendinopathy: a randomized controlled trial with 1-year follow-up. Am J Sports Med 2013;41(11):2609-2616

18 Green S, Buchbinder R, Hetrick S. Physiotherapy interventions for shoulder pain. Cochrane Database Syst Rev 2003;(02):CD004258 
19 Rha DW, Park G-Y, Kim Y-K, Kim MT, Lee SC. Comparison of the therapeutic effects of ultrasound-guided platelet-rich plasma injection and dry needling in rotator cuff disease: a randomized controlled trial. Clin Rehabil 2013;27(02):113-122

20 Buchbinder R, Green S, Youd JM. Corticosteroid injections for shoulder pain. Cochrane Database Syst Rev 2003;(01): CD004016

21 Min KS, St Pierre P, Ryan PM, Marchant BG, Wilson CJ, Arrington ED. A double-blind randomized controlled trial comparing the effects of subacromial injection with corticosteroid versus NSAID in patients with shoulder impingement syndrome. JShoulder Elbow Surg 2013;22(05):595-601

22 Rhon DI, Boyles RB, Cleland JA. One-year outcome of subacromial corticosteroid injection compared with manual physical therapy for the management of the unilateral shoulder impingement syndrome: a pragmatic randomized trial. Ann Intern Med 2014;161(03):161-169

23 Görmeli G, Görmeli CA, Ataoglu B, Çolak C, Aslantürk O, Ertem K. Multiple PRP injections are more effective than single injections and hyaluronic acid in knees with early osteoarthritis: a randomized, double-blind, placebo-controlled trial. Knee Surg Sports Traumatol Arthrosc 2017;25(03):958-965

24 Charousset C, Zaoui A, Bellaiche L, Bouyer B. Are multiple plateletrich plasma injections useful for treatment of chronic patellar tendinopathy in athletes? a prospective study. Am J Sports Med 2014;42(04):906-911

25 Jo CH, Lee SY, Yoon KS, Shin S. Effects of Platelet-Rich Plasma With Concomitant Use of a Corticosteroid on Tenocytes From Degenerative Rotator Cuff Tears in Interleukin $1 \beta$-Induced Tendinopathic Conditions. Am J Sports Med 2017;45(05):1141-1150 